\title{
On Weakly Measurable Functions
}

\author{
by \\ Szymon ŻEBERSKI
}

Presented by Czestaw RYLL-NARDZEWSKI

Summary. We show that if $T$ is an uncountable Polish space, $\mathcal{X}$ is a metrizable space and $f: T \rightarrow \mathcal{X}$ is a weakly Baire measurable function, then we can find a meagre set $M \subseteq T$ such that $f[T \backslash M]$ is a separable space. We also give an example showing that "metrizable" cannot be replaced by "normal".

1. Introduction. It is known that for a partition $\mathcal{A}$ of the real line consisting of sets of Lebesgue measure zero, the union of some of these sets is Lebesgue nonmeasurable. An analogous result is known for sets of the first category (Lebesgue measurability is then replaced by having the Baire property). Actually, this result remains true if in the above statement, the real line is replaced by any Polish space, the $\sigma$-ideals of sets of Lebesgue measure zero or of sets of the first category are replaced by any $\sigma$-ideal $I$ with a Borel base, and instead of assuming that the family $\mathcal{A}$ of sets of an ideal is a partition of the space, we assume that $\mathcal{A}$ is point-finite and its union is not in $I$. The conclusion says now that there exists a subfamily $\mathcal{A}^{\prime}$ of $\mathcal{A}$ whose union is not in the $\sigma$-algebra generated by the $\sigma$-algebra of Borel sets and $I$ (see [1]). This result is called the Four Poles Theorem in the literature.

It is known that within ZFC one cannot replace the assumption that $\mathcal{A}$ is point-finite by $\mathcal{A}$ being point-countable (see [5, p. 64]).

In various cases it is possible to obtain more than nonmeasurability of the union of a subfamily of $\mathcal{A}$. Namely, the intersection of this union with any measurable set that is not in $I$ is nonmeasurable (recall that measurability is understood here in the sense of belonging to the $\sigma$-algebra generated by the family of Borel sets and $I$ ). Such a strong conclusion can be obtained for the

2000 Mathematics Subject Classification: 03E35, 03E75.

Key words and phrases: Baire property, meagre set, measurable function. 
ideal of first Baire category sets under the assumption that $\mathcal{A}$ is a partition, but without assuming anything about the regularity of the elements of $\mathcal{A}$ (see [3]).

The Four Poles Theorem has a variety of applications. Many of them can be found in [2]. Recall that a function $f: \mathbb{R} \rightarrow \mathcal{X}$ is called Baire measurable if for every open set $U \subseteq \mathcal{X}$ the set $f^{-1}[U]$ has the Baire property. In a similar way we can define $\mathcal{B}[I]$-measurable functions (for $I$ as in the Four Poles Theorem). One of the results from [2] says that if $\mathcal{X}$ is a metrizable space and $f: \mathbb{R} \rightarrow \mathcal{X}$ is a $\mathcal{B}[I]$-measurable function, then we can find a set $A \in I$ such that $f[\mathbb{R} \backslash A]$ is separable.

In this paper we show a similar result for a wider class of functions. The proof uses the result from [3]. We also show that in our result the assumption that $\mathcal{X}$ is metrizable cannot be replaced by $\mathcal{X}$ being normal.

2. Definitions and notations. The cardinality of a set $A$ is denoted by $|A|$. Cardinal numbers will usually be denoted by $\kappa$ and $\lambda$. The family of all subsets of cardinality not greater than $\kappa$ of a set $A$ is denoted by $[A] \leq \kappa$. The set of real numbers is denoted by $\mathbb{R}$. An ideal $I$ of subsets of a set $X$ is a family of subsets of $X$ which is closed under finite unions and taking subsets and such that $[X]^{<\omega} \subseteq I$. A family of sets is a $\sigma$-ideal if it is an ideal and is closed under countable unions. For an ideal $I$, the symbol $I^{*}$ denotes its dual filter.

For a topological space $T$, we denote by $\mathcal{O}_{T}$ the family of all open subsets of $T$, and by $\mathcal{B}_{T}$ the family of Borel subsets of $T$. If $I$ is an ideal of subsets of a set $X$ and $\mathcal{S}$ is a field of subsets of $X$, then $\mathcal{S}[I]$ denotes the field generated by $\mathcal{S} \cup I$. If $I$ is also a $\sigma$-ideal and $\mathcal{S}$ is a $\sigma$-field then $\mathcal{S}[I]$ is also a $\sigma$-field.

Let $T$ be an uncountable Polish topological space. The $\sigma$-ideal of all meagre subsets of $T$ is denoted by $\mathbb{K}_{T}$. The $\sigma$-ideal of Lebesgue measure zero subsets of $\mathbb{R}$ will be denoted by $\mathbb{L}$ and the $\sigma$-ideal of sets of the first Baire category in $\mathbb{R}$ will be denoted by $\mathbb{K}$. Then $B_{\mathbb{R}}[\mathbb{L}]$ is the $\sigma$-field of Lebesgue measurable subsets of $\mathbb{R}$ and $B_{\mathbb{R}}[\mathbb{K}]$ is the $\sigma$-field of subsets of $\mathbb{R}$ with the Baire property.

If $I$ is an ideal of subsets of a topological space $T$ then we say that the ideal $I$ has a Borel base if for each set $X \in I$ there exists a set $Y \in \mathcal{B}_{T}$ such that $X \subseteq Y$. The two classical ideals $\mathbb{K}$ and $\mathbb{L}$ have Borel bases.

Let $\mathbb{B}$ be a complete Boolean algebra. We say that $\mathbb{B}$ satisfies c.c.c. (countable chain condition) if every antichain of elements of $\mathbb{B}$ is countable.

For any infinite cardinal $\kappa$ consider the topological sum of the family of spaces $\{[0,1] \times\{\xi\}: \xi<\kappa\}$. Identify in this sum all points $(0, \xi)$, where $\xi<\kappa$, and denote the space obtained by $J_{\kappa}$. It is well known that any metrizable space can be embedded into a countable product of spaces $J_{\kappa_{n}}$ (see [4]). 
If $\mathcal{X}$ is a metrizable space and $\mathcal{A}$ is an open cover of $\mathcal{X}$ then, by Stone's theorem, we can find a family $\left\{\mathcal{A}_{n}\right\}_{n \in \omega}$ such that $\bigcup_{n \in \omega} \mathcal{A}_{n}$ is a point-finite open cover of $\mathcal{X}$ and each $\mathcal{A}_{n}$ is a family of pairwise disjoint open sets and each element of $\mathcal{A}_{n}$ is contained in some element of $\mathcal{A}$. This result can be found in [4].

3. Weakly measurable functions. In this section $T$ denotes an uncountable Polish space. Recall the following definition.

Definition 3.1. Let $N \subseteq X \subseteq T$. We say that the set $N$ is completely Baire nonmeasurable in $X$ if

$$
\left(\forall A \in \mathcal{B}_{T}\right)\left(A \cap X \notin \mathbb{K}_{T} \rightarrow\left(A \cap N \notin \mathbb{K}_{T}\right) \wedge\left(A \cap(X \backslash N) \notin \mathbb{K}_{T}\right)\right) .
$$

In [3] we can find the following theorem.

THEOREM 3.2. Let $\mathcal{P}$ be a pairwise disjoint family of meagre sets such that $\bigcup \mathcal{P} \notin \mathbb{K}_{T}$. Then there exists a subfamily $\mathcal{P}^{\prime} \subseteq \mathcal{P}$ such that $\bigcup \mathcal{P}^{\prime}$ is completely Baire nonmeasurable in $\bigcup \mathcal{P}$.

Definition 3.3. Let $I \subseteq P(T)$ be a $\sigma$-ideal with a Borel base such that the Boolean algebra $\mathcal{B}_{T} / I$ satisfies c.c.c. Let $A$ be any subset of $T$. We denote by $[A]_{I}$ the Borel envelope of $A$, i.e. the minimal (in the sense of the algebra $\left.\mathcal{B}_{T} / I\right)$ Borel set containing $A$.

The set $[A]_{I}$ is well defined since the algebra $\mathcal{B}_{T} / I$ satisfies c.c.c. To find $[A]_{I}$, take the maximal antichain $\mathcal{A}$ of $I$-positive Borel sets which are disjoint from $A$. The family $\mathcal{A}$ is countable. So, its union $\bigcup \mathcal{A}$ is a Borel set. The complement $(\bigcup \mathcal{A})^{\mathrm{c}}$ is the required envelope.

Definition 3.4. Let $\mathcal{X}$ be a topological space and $f: T \rightarrow \mathcal{X}$ be a function. We say that $f$ is weakly Baire measurable if

$$
\begin{aligned}
\left(\forall U, V \in \mathcal{O}_{\mathcal{X}}\right)\left(U \cap V=\emptyset \wedge f^{-1}[U] \notin \mathbb{K}_{T}\right. & \wedge f^{-1}[V] \notin \mathbb{K}_{T} \\
& \left.\rightarrow\left[f^{-1}[U]\right]_{\mathbb{K}_{T}} \neq\left[f^{-1}[V]\right]_{\mathbb{K}_{T}}\right) .
\end{aligned}
$$

First, notice that every Baire measurable function $f: T \rightarrow \mathcal{X}$ is weakly Baire measurable. Indeed, if we have two disjoint open sets $U, V \subseteq \mathcal{X}$ such that $f^{-1}[U] \notin \mathbb{K}_{T}$ and $f^{-1}[V] \notin \mathbb{K}_{T}$ then since the latter sets are disjoint and have the Baire property, $\left[f^{-1}[U]\right]_{\mathbb{K}_{T}} \cap\left[f^{-1}[V]\right]_{\mathbb{K}_{T}} \in \mathbb{K}_{T}$. This implies that the condition of weak Baire measurability is satisfied.

On the other hand, we can easily find a function $f: \mathbb{R} \rightarrow \mathbb{R}$ which is Lebesgue measurable but not weakly Baire measurable. Namely, consider a partition $\mathbb{R}=K \cup L$ such that $L \in \mathbb{L}$ and $K \in \mathbb{K}$. Fix any partition $L=L_{1} \cup L_{2}$ such that $\left[L_{1}\right]_{\mathbb{K}}=\left[L_{2}\right]_{\mathbb{K}}=[\mathbb{R}]_{\mathbb{K}}$. The characteristic function of $L_{1}$ is Lebesgue measurable but not weakly Baire measurable.

Recall the following well known result due to Banach (see [6]). 
TheOREM 3.5 (Banach). Let $\mathcal{X}$ be any topological space. Consider the family

$$
\mathcal{A}=\left\{U \in \mathcal{O}_{\mathcal{X}}: U \text { is meagre in } \mathcal{X}\right\} .
$$

Then $\bigcup \mathcal{A}$ is also meagre in $\mathcal{X}$.

The next theorem is similar:

TheOREM 3.6. Let $\mathcal{X}$ be any metrizable space. Let $f: T \rightarrow \mathcal{X}$ be a weakly Baire measurable function. Consider the family

$$
\mathcal{A}=\left\{f^{-1}[U] \in \mathbb{K}_{T}: U \in \mathcal{O}_{\mathcal{X}}\right\} .
$$

Then $\bigcup \mathcal{A}$ is also meagre in $T$.

Proof. Let $\widetilde{\mathcal{A}}=\left\{U \in \mathcal{O}_{X}: f^{-1}[U] \in \mathbb{K}_{T}\right\}$. Then $\bigcup \mathcal{A}=f^{-1}[\bigcup \widetilde{\mathcal{A}}]$. Since $\mathcal{X}$ is metrizable, $\bigcup \widetilde{\mathcal{A}}$ is also metrizable. So, we can find families $\widetilde{\mathcal{A}}_{n}(n \in \omega)$ satisfying the following conditions:

- $\bigcup_{\widetilde{\mathcal{A}}} \widetilde{\mathcal{A}}=\bigcup_{n \in \omega} \bigcup \widetilde{\mathcal{A}}_{n}$

- $\widetilde{\mathcal{A}}_{n}$ is a family of pairwise disjoint open sets,

- $\left(\forall U \in \widetilde{\mathcal{A}}_{n}\right)(\exists V \in \widetilde{\mathcal{A}})(U \subseteq V)$.

From the above conditions it follows that $\widetilde{\mathcal{A}}_{n} \subseteq \widetilde{\mathcal{A}}$ for each $n \in \omega$. Define

$$
\mathcal{A}_{n}=\left\{f^{-1}[U]: U \in \widetilde{\mathcal{A}}_{n}\right\} .
$$

We claim that $\cup \mathcal{A}_{n} \in \mathbb{K}_{T}$. Suppose otherwise. Then each $\mathcal{A}_{n}$ satisfies the assumption in Theorem 3.2. Hence we can find two disjoint open sets $U_{0}, U_{1} \subseteq \mathcal{X}$ such that $f^{-1}\left[U_{0}\right]$ and $f^{-1}\left[U_{1}\right]$ are completely Baire nonmeasurable in $\bigcup \mathcal{A}_{n}$. In particular, $\left[f^{-1}\left[U_{0}\right]\right]_{\mathbb{K}_{T}}=\left[f^{-1}\left[U_{1}\right]\right]_{\mathbb{K}_{T}}$, which contradicts the weak Baire measurability of $f$.

Notice that $\bigcup \mathcal{A}=\bigcup_{n \in \omega} \cup \mathcal{A}_{n}$. So, $\cup \mathcal{A} \in \mathbb{K}_{T}$.

THEOREM 3.7. Let $I \subseteq P(T)$ be a $\sigma$-ideal with a Borel base such that $\mathcal{B}_{T} / I$ satisfies c.c.c. Let $\left\{A_{\xi}: \xi \in \omega_{1}\right\} \subseteq P(T)$ be any family. Then we can find two disjoint countable subsets $B, C$ of $\omega_{1}$ such that $\left[\bigcup_{\xi \in B} A_{\xi}\right]_{I}=$ $\left[\bigcup_{\xi \in C} A_{\xi}\right]_{I}$.

Proof. For $\alpha<\beta<\omega_{1}$ let

$$
A_{\alpha}^{\beta}=\bigcup\left\{A_{\xi}: \alpha<\xi<\beta\right\} .
$$

Proceeding by transfinite induction, we construct a sequence $\left\{\alpha_{\xi}: \xi<\omega_{1}\right\}$ of ordinals less than $\omega_{1}$ such that

$$
\left(\forall \xi, \zeta, \eta \in \omega_{1}\right)\left(\xi<\zeta<\eta \rightarrow\left[A_{\alpha_{\xi}}^{\alpha_{\zeta}}\right]_{I}=\left[A_{\alpha_{\xi}}^{\alpha_{\eta}}\right]_{I}\right) \text {. }
$$

In step $\beta$ consider the sequence $\left\{\left[A_{\alpha_{\beta}}^{\alpha}\right]_{I}: 0<\alpha<\omega_{1}\right\}$, which is increasing. Since $\mathcal{B}_{T} / I$ satisfies c.c.c., this sequence is constant from some $\gamma$ on. Put $\alpha_{\beta+1}=\gamma$. 
Now, consider the sequence $\left\{\left[A_{\alpha_{\xi}}^{\alpha_{\xi+1}}\right]_{I}: \xi<\omega_{1}\right\}$, which is decreasing. Since $\mathcal{B}_{T} / I$ satisfies c.c.c., this sequence is constant from some $\gamma<\omega_{1}$ on. Put

$$
B=\left(\alpha_{\gamma}, \alpha_{\gamma+1}\right), \quad C=\left(\alpha_{\gamma+1}, \alpha_{\gamma+2}\right) .
$$

These sets satisfy the required condition.

TheOREM 3.8. Let $\mathcal{X}$ be a metrizable space. Let $f: T \rightarrow \mathcal{X}$ be a weakly Baire measurable function. Then there is a meagre set $M \subseteq T$ such that $f[T \backslash M]$ is separable.

Proof. Consider the family $\mathcal{A}=\left\{f^{-1}[U] \in \mathbb{K}_{T}: U \in \mathcal{O}_{\mathcal{X}}\right\}$. By Theorem 3.6 we know that $\bigcup \mathcal{A} \in \mathbb{K}_{T}$. Define $M=\bigcup \mathcal{A}$.

As $f[T \backslash M]$ is metrizable, we can treat it as a subspace of $\prod_{n \in \omega} J_{\kappa_{n}}$. Assume that each $\kappa_{n}$ is the least possible cardinal.

We will show that $\kappa_{n} \leq \omega$ for each $n \in \omega$. Suppose otherwise. Without loss of generality we can assume that $\kappa_{0}=\omega_{1}$. This means that there exists a family $\left\{U_{\alpha}: \alpha<\omega_{1}\right\}$ of nonempty pairwise disjoint open sets in $f[T \backslash M]$. The family $\left\{f^{-1}\left[U_{\alpha}\right]: \alpha<\omega_{1}\right\}$ satisfies the condition $f^{-1}\left[U_{\alpha}\right] \notin \mathbb{K}_{T}$ for all $\alpha$. Applying Theorem 3.7 to this family, we get a contradiction to weak measurability of $f$. So, $\kappa_{n} \leq \omega$ for each $n \in \omega$. This means that $f[T \backslash M] \subseteq$ $\prod_{n \in \omega} J_{\omega}$, which implies that $f[T \backslash M]$ is separable.

4. Nonmetrizable spaces. Now, let us show that Theorems 3.6 and 3.8 do not hold if we replace "metrizable" by "normal". In fact, we will work with the space $\omega_{1}$ equipped with the order topology.

Note that we cannot hope to find a counterexample in ZFC, because in some models the Four Poles Theorem or even its stronger version about completely nonmeasurable unions holds without any assumptions on the given family of small sets. In such models Theorems 3.6 and 3.8 hold for any topological space $\mathcal{X}$.

Therefore our considerations will take place in a particular model of ZFC. Namely, let us start with a model $V$ which satisfies GCH. We can extend it to a model $V^{\prime}$ by adding $\omega_{2}$ random reals. The universe $V^{\prime}$ is good enough for what we need. Notice that in $V^{\prime}$ we have $2^{\omega}=2^{\omega_{1}}=\omega_{2}$.

Let us start with an easy observation.

Claim 4.1. In $V^{\prime}$ there exists a family $\left\{K^{\alpha}\right\}_{\alpha \in \omega_{2}} \subseteq \mathbb{K}$ such that for every uncountable set $A \subseteq \omega_{2}$ we have $\bigcup_{\alpha \in A} K^{\alpha}=\mathbb{R}$.

Proof. Let $\left\{r_{\alpha}\right\}_{\alpha \in \omega_{2}}$ be the family of generic random reals. Fix a partition of $\mathbb{R}$ into a $G_{\delta}$ set of Lebesgue measure zero and an $F_{\sigma}$ set of first Baire category, $\mathbb{R}=K \cup L$, where $K \in \mathbb{K}$ and $L \in \mathbb{L}$. Put

$$
K^{\alpha}=K+r_{\alpha}=\left\{k+r_{\alpha}: k \in K\right\} .
$$


Let $A \subseteq \omega_{2}$ be uncountable. Assume that there is a real number $x \in \mathbb{R}$ satisfying $x \notin \bigcup_{\alpha \in A} K^{\alpha}$. So, if $\alpha \in A$ then $x \notin r_{\alpha}+K$. Thus $r_{\alpha} \notin x-K=$ $\{x-k: k \in K\}$. The set $x-K$ is meager. The real number $x$ belongs to $V\left[r_{\alpha}: \alpha \in I\right]$, where $I$ is a countable subset of $\omega_{2}$. Since $A$ is uncountable, there exists $\xi \in A \backslash I$. So, $r_{\xi}$ is a random real over $V\left[r_{\alpha}: \alpha \in I\right]$. But the set $(x-K)^{\mathrm{c}}$ is in $\mathbb{L}$ and is coded in the model $V\left[r_{\alpha}: \alpha \in I\right]$. Moreover, $r_{\xi} \in(x-K)^{\mathrm{c}}$, which gives a contradiction.

The next claim is a generalization of the result presented in [5, p. 64].

Claim 4.2. In $V^{\prime}$ the following statement holds. Fix any $\sigma$-ideal $\mathcal{I} \subseteq$ $P\left(\omega_{1}\right)$. There exists a point-countable family $\left\{\widetilde{K}^{\alpha}\right\}_{\alpha \in \omega_{1}}$ of meagre subsets of the real line such that for every set $A \subseteq \omega_{1}$ we have

$$
\begin{aligned}
& A \in \mathcal{I} \rightarrow \bigcup_{\alpha \in A} \widetilde{K}^{\alpha} \in \mathbb{K}, \\
& A \notin \mathcal{I} \rightarrow \bigcup_{\alpha \in A} \widetilde{K}^{\alpha} \in \mathbb{K}^{*} .
\end{aligned}
$$

Proof. Fix a family $\left\{K^{\alpha}\right\}_{\alpha \in \omega_{2}}$ as in Claim 4.1 and fix a bijection $\phi$ : $P\left(\omega_{1}\right) \rightarrow \omega_{2}$. The required family $\left\{\widetilde{K}^{\alpha}\right\}_{\alpha \in \omega_{1}}$ will have the following properties:

(1) $\left(\forall \alpha<\omega_{1}\right)\left(\widetilde{K}^{\alpha} \subseteq K^{\alpha}\right)$,

(2) $(\forall A \in \mathcal{I})\left(\bigcup\left\{\widetilde{K}^{\alpha}: \alpha \in A\right\} \subseteq K^{\phi(A)}\right)$,

(3) $(\forall A \notin \mathcal{I})\left(\bigcup\left\{\widetilde{K}^{\alpha}: \alpha \in A\right\} \cup K^{\phi(A)}=\mathbb{R}\right)$.

To construct a family satisfying the above conditions, we write them in the following form:

(1) $(\forall x \in \mathbb{R})(\forall A \in \mathcal{I})\left(x \notin K^{\phi(A)} \rightarrow x \notin \bigcup\left\{\widetilde{K}^{\alpha}: \alpha \in A\right\}\right)$,

(2) $(\forall x \in \mathbb{R})(\forall A \notin \mathcal{I})\left(x \notin K^{\phi(A)} \rightarrow x \in \bigcup\left\{\widetilde{K}^{\alpha}: \alpha \in A\right\}\right)$.

Using Claim 4.1 we deduce that for each $x \in \mathbb{R}$ there are only countably many $A \subseteq \omega_{1}$ such that $x \notin K^{\phi(A)}$. Let

$$
\begin{aligned}
\mathcal{I}(x) & =\left\{A \in \mathcal{I}: x \notin K^{\phi(A)}\right\}, \\
\mathcal{I}^{+}(x) & =\left\{A \notin \mathcal{I}: x \notin K^{\phi(A)}\right\} .
\end{aligned}
$$

Let $B(x)=\bigcup \mathcal{I}(x)$. Then $B(x) \in \mathcal{I}$ and for each $A \in \mathcal{I}^{+}(x)$, we have $A \backslash B(x) \notin \mathcal{I}$. In particular $A \backslash B(x) \neq \emptyset$. So, it is possible to ensure that

$$
(\forall x)\left(\forall A \in \mathcal{I}^{+}(x)\right)(\exists \alpha \in A \backslash B(x))\left(x \in \widetilde{K}^{\alpha}\right) .
$$

This finishes the construction of the required family.

The next result is of independent interest. 
Claim 4.3 (Cichoń). In $V^{\prime}$ the following statement holds. Fix any $\sigma$-ideal $\mathcal{I} \subseteq P\left(\omega_{1}\right)$. Then there exists a function $g: \mathbb{R} \rightarrow \omega_{1}$ such that

$$
\left(\forall A \subseteq \omega_{1}\right)\left(A \in \mathcal{I} \leftrightarrow g^{-1}[A] \in \mathbb{K}\right) .
$$

Proof. Let $\left\{\widetilde{K}^{\alpha}\right\}_{\alpha \in \omega_{1}}$ be as in Claim 4.2. Define

$$
\widetilde{K}=\bigcup\left\{\widetilde{K}^{\alpha} \times\{\alpha\}: \alpha \in \omega_{1}\right\} .
$$

Fix an enumeration $\left\{A_{\alpha}: \alpha<\omega_{2}\right\}$ of the $\mathcal{I}$-positive subsets of $\omega_{1}$. Fix also an enumeration $\left\{M_{\alpha}: \alpha<\omega_{2}\right\}$ of the meagre subsets of $\mathbb{R}$ and an enumeration $\left\{x_{\alpha}: \alpha<\omega_{2}\right\}$ of all real numbers. We will construct an increasing sequence $\left\{g_{\alpha}\right\}_{\alpha<\omega_{2}}$ of functions by transfinite induction. In step $\alpha$ we will construct a function $g_{\alpha}$ satisfying the following conditions:

(1) $g_{\alpha} \subseteq \widetilde{K}$

(2) $x_{\alpha} \in \operatorname{dom}\left(g_{\alpha}\right)$,

(3) $\bigcup_{\xi<\alpha} g_{\xi} \subseteq g_{\alpha}$

(4) $\left|g_{\alpha}\right| \leq \omega_{1}$

(5) $(\forall \xi, \zeta<\alpha)(\exists x \in \mathbb{R})\left(x \notin M_{\zeta} \wedge g(x) \in A_{\xi}\right)$.

So, at step $\alpha$, we have to add to $\bigcup_{\xi<\alpha} g_{\xi}$ at most $\omega_{1}$ new points of our function $g$. Since $|\mathbb{R}|=\omega_{2}$, this is possible. Put $g=\bigcup_{\alpha \in \omega_{2}} g_{\alpha}$. This is the required function.

Now, we are able to formulate the result which shows that Theorem 3.6 cannot be generalized too much.

THEOREM 4.4. In $V^{\prime}$ the following statement holds. Let $\tau_{\text {order }}$ be the order topology on $\omega_{1}$. There is a function $f: \mathbb{R} \rightarrow\left(\omega_{1}, \tau_{\text {order }}\right)$ satisfying the following conditions:

(1) $f$ is Baire measurable,

(2) $\bigcup\left\{f^{-1}[U] \in \mathbb{K}: U \in \tau_{\text {order }}\right\}=\mathbb{R}$.

Proof. Applying Claim 4.3 to the ideal $\mathcal{N S}_{\omega_{1}}$ of all nonstationary subsets of $\omega_{1}$, we obtain a function $f: \mathbb{R} \rightarrow \omega_{1}$. This function is Baire measurable. Indeed, take any open subset $U$ of $\omega_{1}$. There are two possibilities.

If $U^{\mathrm{c}}$ is unbounded, then since it is closed, $U \in \mathcal{N} \mathcal{S}_{\omega_{1}}$ and $f^{-1}[U] \in \mathbb{K}$. If $U^{\mathrm{c}}$ is bounded, then $U^{\mathrm{c}} \in \mathcal{N} \mathcal{S}_{\omega_{1}}$ and $f^{-1}\left[U^{\mathrm{c}}\right] \in \mathbb{K}$. So $f^{-1}[U] \in \mathbb{K}^{*}$. In both cases we get a set with the Baire property.

Since every point in $\omega_{1}$ can be covered by an open set which is nonstationary, we have $\bigcup\left\{f^{-1}[U] \in \mathbb{K}: U \in \tau_{\text {order }}\right\}=\mathbb{R}$.

It can be easily seen that the function obtained in Theorem 4.4 is also a counterexample to a generalization of Theorem 3.8 to functions with images in normal topological spaces. 


\section{References}

[1] J. Brzuchowski, J. Cichoń, E. Grzegorek and C. Ryll-Nardzewski, On the existence of nonmeasurable unions, Bull. Polish Acad. Sci. Math. 27 (1979), 447-448.

[2] J. Cichoń and A. Kharazishvili, On ideals with projective bases, Georgian Math. J. 9 (2002), 472-561.

[3] J. Cichoń, M. Morayne, R. Rałowski, C. Ryll-Nardzewski and S. Żeberski, On nonmeasurable unions, to appear.

[4] R. Engelking, General Topology, PWN, Warszawa, 1985.

[5] D. H. Fremlin, Measure-additive coverings and measurable selectors, Dissertationes Math. 260 (1987).

[6] A. S. Kechris, Classical Descriptive Set Theory, Grad. Texts in Math. 156, Springer, 1994.

Szymon Żeberski

Institute of Mathematics

University of Wrocław

Pl. Grunwaldzki 2/4

50-384 Wrocław, Poland

E-mail: szebe@math.uni.wroc.pl

Received October 12, 2005;

received in final form January 11, 2006 